Papers and Proceedings of the Royal Society of Tasmania, Vol. 110, 1976

(ms, received 23.2, 1976)

\title{
A SUMMARY OF THE OLDEST AGES FOR THE WORLD'S ISLANDS
}

\author{
by Oakley Shields \\ University of Tasmania
}

\section{ABSTRACT}

The oldest ages of rocks on the world's islands are sumnarized (0ligocene or older), based on published literature. All the oceans and perhaps all the seas contain a few continental islands, indicative of debris left from continental rifting prior to ocean basin formation by seafloor spreading. The Musicians Seamounts and the Hawaiian Ridge (both Cretaceous), and the Tuamotu Islands (Eocene) are the same age as the surrounding seafloor and probably formed at the crest of the East Pacific Rise. Future drilling and dredging operations are expected to modify and add to this list, as recent volcanism may well have obscured the much older basement rocks on many islands. Thus, the faunas of the Galapagos, Mauritius, Rodriguez, and Tristan da Cunha suggest they are continental islands, while their known surface geology yields dates of only $8 \mathrm{m.y}$. or less.

\section{INTRODUCTION}

The following is a summary of the oldest ages (i.e. Oligocene or older) of rocks on the world's islands (including some seamounts, guyots, and sunken plateaus), compiled from the literature. Especially interesting from tectonic and biogeographic considerations are islands with continental rock-types. "'Continental rocks'include plutonic rocks such as granite and diorite and metamorphic rocks such as schist and slate. As a general rule such rocks are not found except on continents or on islands obviously once connected with continents. The deep-seated plutonic rocks are exposed only after prolonged erosion and most islands have not suffered sufficient erosion even if it be assumed that they have a plutonic core. The metamorphic rocks demand powerful earth movements and these cannot be developed on tiny land masses. Hence, the occurrence of either plutonic or metamorphic rocks on an island suggests that it once was a part of a much larger land mass. The 'island rocks' consist largely of volcanic rocks and limestones and sediments derived from these" (Wentworth \& Ladd 1931, p.6). According to Daly (1916a), Pacific islands having rock types characteristic of large areas in the continents include granite, gneiss, schist, serpentine, and deformed sediments (However, more recent work suggests granitic metamorphism requires diapiric transport up from significant depths and is not excluded from islands (Carey pers. com.); serpentine is known to occur along some transform faults, and mid-ocean ridges (Chernysheva 1971), and it has been reported from the Puerto Rico Trench by Perfit, Heezen, and Rawson 1974.) Schists can occur in shear-zones. A continental flora or fauna means that that island resembles a continent in aspect rather than an oceanic island - i.e., the biota is not markedly impoverished, or forms are present such as land turtles, amphibians, flightless birds or the like which suggest past contacts with a continent. The distinction between oceanic and continental islands was first made by Charles Darwin; oceanic islands are volcanic or coral and lack native mammals and amphibians (Wa1lace 1887). 


\section{OLDEST AGES FOR ISLANDS}

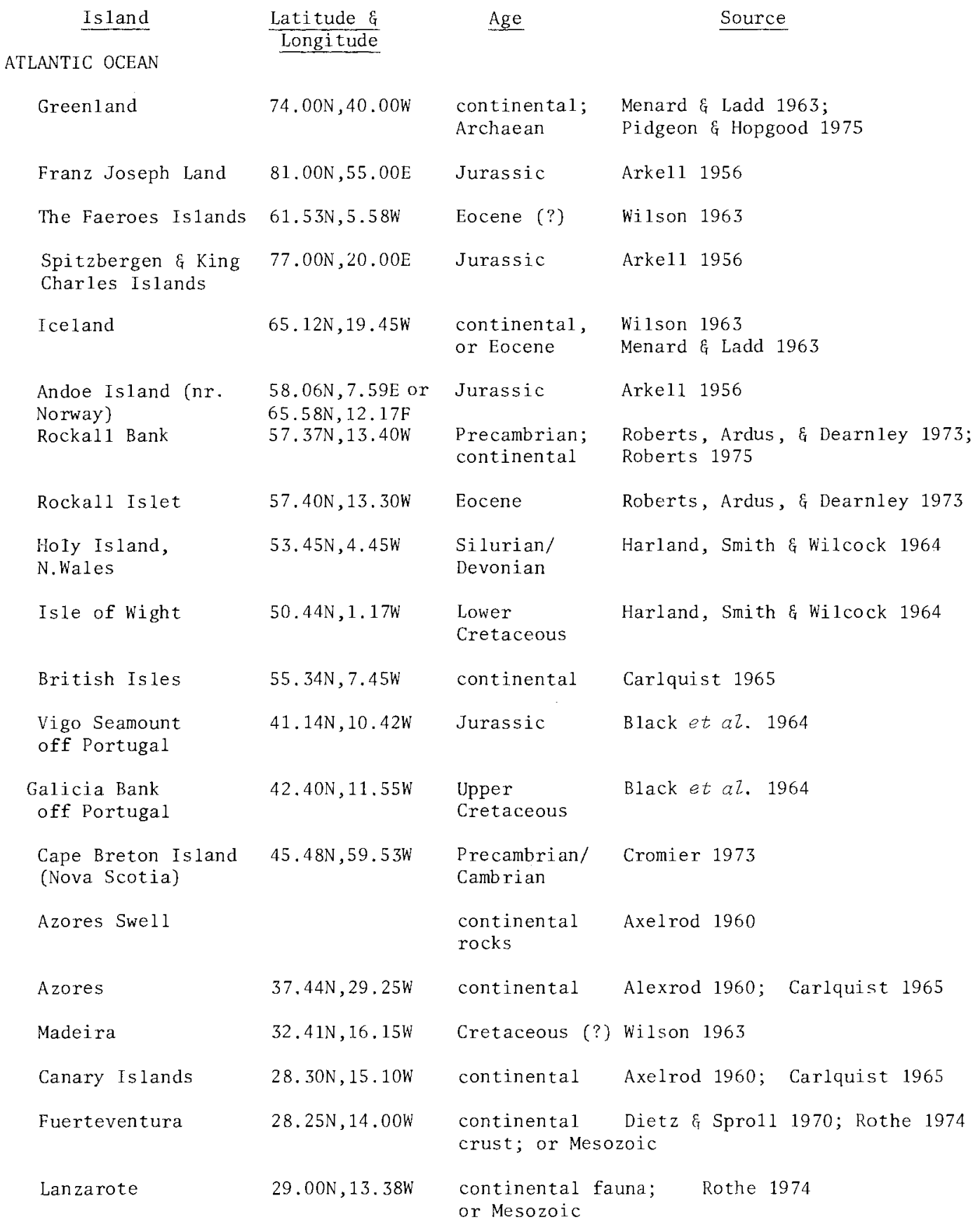




\begin{tabular}{|c|c|c|c|}
\hline Island & $\frac{\text { Latitude E }}{\text { Longitude }}$ & Age & Source \\
\hline Tenerife & $28.15 \mathrm{~N}, 16.35 \mathrm{~W}$ & continental fauna & Rothe 1974 \\
\hline Cape Verde Islands & $16.00 \mathrm{~N}, 24.00 \mathrm{~W}$ & continental & Carlquist 1965 \\
\hline Maio I. & $15.14 \mathrm{~N}, 23.10 \mathrm{~W}$ & Upper Jurassic & $\begin{array}{r}\text { Smith 1971; Mitche11-Thome } \\
1974\end{array}$ \\
\hline Sao Nicolao Islands & $16.35 \mathrm{~N}, 24.15 \mathrm{~W}$ & Upper Jurassic (?) & Mitchel1-Thome 1974 \\
\hline Conception Bank & $23.55 \mathrm{~S}, 14.30 \mathrm{E}$ & continental crust & Dietz \& Sprol1 1970 \\
\hline Fernando Poo & $3.22 \mathrm{~N}, 7.37 \mathrm{E}$ & Cretaceous (?) & Mitche11-Thome 1970 \\
\hline Principe & $1.42 \mathrm{~N}, 5.38 \mathrm{E}$ & $\begin{array}{c}\text { end of Cretaceous } \\
\text { (?) }\end{array}$ & Mitchel1-Thome 1970 \\
\hline Sao Tome & $0.41 \mathrm{~N}, 6.01 \mathrm{E}$ & $\begin{array}{l}\text { Lower } \\
\text { Cretaceous (?) }\end{array}$ & Mitche11-Thome 1970 \\
\hline Annobon & $2.00 \mathrm{~S}, 3.30 \mathrm{E}$ & Cretaceous (?) & Mitche 11 -Thome 1970 \\
\hline Bermuda & $32.18 \mathrm{~N}, 64.45 \mathrm{~W}$ & $\begin{array}{l}\text { Eocene, pre- } \\
\text { Eocene, or } \\
\text { Triassic (?) }\end{array}$ & $\begin{array}{l}\text { Reed 1949; Wilson 1963; } \\
\text { Engelen 1964, Reynolds } 1973\end{array}$ \\
\hline Bahamian Platform & & $\begin{array}{l}\text { Jurassic; or } \\
\text { Palaeozoic (?) }\end{array}$ & Lynts 1970 \\
\hline Bahamas & $26.15 \mathrm{~N}, 76.00 \mathrm{~W}$ & Early Cretaceous & Wilson 1963 \\
\hline $\begin{array}{l}\text { Demerara Plateau } \\
\text { (off South America) }\end{array}$ & $9.26 \mathrm{~N}, 54.06 \mathrm{~W}$ & $\begin{array}{l}\text { Late Jurassic/ } \\
\text { Ear1y Cretaceous }\end{array}$ & Fox, Heezen, E Johnson 1970 \\
\hline $\begin{array}{l}\text { St. Peter \& St.Paul } \\
\text { Rocks }\end{array}$ & $0.56 \mathrm{~N}, 29.22 \mathrm{~W}$ & $4550+\quad$ m.y. & Wright 1965 \\
\hline Fernando de Noronha & $3.50 \mathrm{~S}, 33.15 \mathrm{~W}$ & Upper Cretaceous & Mitche11-Thome 1970 \\
\hline Ascension & $8.00 \mathrm{~S}, 13.00 \mathrm{~W}$ & $\begin{array}{c}\text { continenta } 1 \text { rocks } \\
\text { (?) }\end{array}$ & $\begin{array}{l}\text { Alexrod 1960; Carlquist } \\
\text { 1965; Mi tche11-Thome } 1970\end{array}$ \\
\hline St. Helena & $16.01 \mathrm{~S}, 5.16 \mathrm{~W}$ & Eocene & Mitche11-Thome 1970 \\
\hline Walvis Ridge & & continental rocks & Axe1rod 1960 \\
\hline Trinidade & $21.00 \mathrm{~S}, 32.00 \mathrm{~W}$ & continental rocks & Axe1rod 1960 \\
\hline Martin Vaz & $20.30 \mathrm{~S}, 28.52 \mathrm{~W}$ & Upper Cretaceous & Mitche 11 -Thome 1970 \\
\hline Tristan da Cunha & $35.30 \mathrm{~S}, 12.15 \mathrm{~W}$ & continenta 1 & Axelrod 1960; Car1quist 1965 \\
\hline Meteor Bank & & continental rocks & Axelrod 1960 \\
\hline
\end{tabular}




\begin{tabular}{|c|c|c|c|}
\hline Island & $\frac{\text { Latitude } \&}{\text { Longitude }}$ & Age & Source \\
\hline Falkland Is lands & $50.45 S, 61.00 \mathrm{~W}$ & Precambrian & Mitche11-Thome 1970 \\
\hline $\begin{array}{l}\text { is lands of the North } \\
\xi \text { South Scotia Ridges }\end{array}$ & & $\begin{array}{l}\text { continental rocks } \\
\text { (?Palaeozoic or } \\
\text { older) }\end{array}$ & Dalziel 1972 \\
\hline South Georgia & $54.00 \mathrm{~S}, 37.00 \mathrm{~W}$ & $\begin{array}{l}\text { continenta1; or } \\
\text { Jurassic/ } \\
\text { Cretaceous }\end{array}$ & $\begin{array}{l}\text { Axelrod 1960; Carlquist } 1965 \text {; } \\
\text { E11iot } 1975\end{array}$ \\
\hline South Orkneys & $60.38 \mathrm{~S}, 45.30 \mathrm{~W}$ & $\begin{array}{l}\text { continental } \\
\text { Precambrian }\end{array}$ & $\begin{array}{l}\text { Alexrod 1960; Carlquist } 1965 \text {; } \\
\text { Elliot } 1975\end{array}$ \\
\hline Signy I. & $60.43 \mathrm{~S}, 45.36 \mathrm{~W}$ & $176-199 \mathrm{~m} . \mathrm{y}$. & Alexrod 1960; Carlquist 1965 \\
\hline Moe $I$. & $60.45 S, 45.41 \mathrm{~W}$ & $176-199 \mathrm{~m} \cdot y$. & Axe1rod 1960; Carlquist 1965 \\
\hline South Shet lands & $62.00 \mathrm{~S}, 58.00 \mathrm{~W}$ & $\begin{array}{l}\text { Continental; } \\
\text { or Late } \\
\text { Palaeozoic }\end{array}$ & $\begin{array}{l}\text { Axelrod 1960; Carlquist } 1965 \text {; } \\
\text { El1iott } 1975\end{array}$ \\
\hline A1exander Is 1 and & $71.00 \mathrm{~S}, 71.00 \mathrm{~W}$ & Late Palaeozoic & E11iot 1975 \\
\hline $\begin{array}{l}\text { Northern Antarctic } \\
\text { Peninsula }\end{array}$ & & Middle Palaeozoic & Elliot 1975 \\
\hline DITERRANEAN SEA & & & \\
\hline Gilbra1tar & $36.08 \mathrm{~N}, 5.22 \mathrm{~W}$ & Jurassic (?) & Reed 1949 \\
\hline Minorca & $40.00 \mathrm{~N}, 4.00 \mathrm{E}$ & Palaeozoic & Arke11 1956 \\
\hline Mallorca (Majorca) & $39.30 \mathrm{~N}, 3.00 \mathrm{E}$ & Palaeozoic & Arkell 1956 \\
\hline Corsica & $42.10 \mathrm{~N}, 8.55 \mathrm{E}$ & Upper Pa1aeozoic & Arke11 1956; Klenme 1958 \\
\hline Sardinia & $40.08 \mathrm{~N}, 9.05 \mathrm{E}$ & Lower Palaeozoic & Arke11 1956; Klenme 1958 \\
\hline Is land of Elba & $42.42 \mathrm{~N}, 10.25 \mathrm{E}$ & Upper Jurassic & Grunau 1965 \\
\hline Sicily & $37.38 \mathrm{~N}, 13.30 \mathrm{E}$ & Upper Palaeozoic & Klemme 1958 \\
\hline Maltese Islands & $36.00 \mathrm{~N}, 14.15 \mathrm{E}$ & Oligocene & Reed 1949 \\
\hline Crete & $35.15 \mathrm{~N}, 24.30 \mathrm{E}$ & Upper Palaeozoic & Klemme 1958 \\
\hline Cyprus & $34.56 \mathrm{~N}, 31.28 \mathrm{E}$ & Upper Palaeozoic & Reed 1949; K1emme 1958 \\
\hline Ionian Isles & $39.10 \mathrm{~N}, 20.05 \mathrm{E}$ & Jurassic & Arke11 1956 \\
\hline
\end{tabular}




\section{Is 1and}

INDIAN OCEAN

Bahrain Is 1ands

(Persian Gulf)

Socotra

Abd-E1-Kuri

Ceylon

Laccadive, Ma1dive,

$\&$ Chagos Islands

Seyche1les

Mahe

Silhouette Is.

Praslin

Northern end of the

Mascarene Ridge \&

Cargados Carajos

Shoals

Mauritius

Providence

Comores Islands

Madagascar

St. Paul

Kerguelen

Heard Island

Crozet (Possession) 46.20S, 51.30E Is land

$\frac{\text { Latitude \& }}{\text { Longitude }}$
$5.20 \mathrm{~S}, 55.10 \mathrm{E}$

$4.41 \mathrm{~S}, 55.30 \mathrm{E}$

$9.14 \mathrm{~S}, 51.02 \mathrm{E}$

$11.46 \mathrm{~S}, 44.12 \mathrm{E}$

$53.10 \mathrm{~S}, 74.35 \mathrm{E}$

$26.15 \mathrm{~N}, 51.17 \mathrm{E}$

$13.00 \mathrm{~N}, 52.30 \mathrm{E}$

$12.21 \mathrm{~N}, 51.00 \mathrm{E}$

$8.45 N, 82.30 \mathrm{E}$

$20.18 \mathrm{~S}, 57.36 \mathrm{E}$

$21.30 \mathrm{~S}, 46.00 \mathrm{E}$

$38.43 \mathrm{~S}, 77.31 \mathrm{E}$

$49.50 \mathrm{~S}, 69.30 \mathrm{E}$

$46.20 \mathrm{~S}, 51.30 \mathrm{E}$

Age

Source

Cretaceous

Reed 1949

continenta1;

Archaean

Archaean

Precambrian

continenta 1

crust (?)

Precambrian

$645 \pm 55$ m.y.

$62 \pm 12 \mathrm{~m} . \mathrm{y}$.

$647 \pm 55$ m.y.

continental crust Matthews \& Davies 1966

continental rocks; de Haga Haig 1895;

or Cretaceo-

Tertiary

Axelrod 1960; Carlquist

1965; Simpson 1950

Eocene/0ligocene

continental rocks

Reed 1949; Wilson 1963

Axelrod 1960; Carlquist 1965; Flower \& Strong 1969

(but see Wright \& McCurry 1970)

\section{Precambrian}

Wilson 1963

continental rocks Axelrod 1960; Carlquist

1965

continental rocks; Axelrod 1960; Carlquist or Upper Cretaceous-Palaeocene

1965; Nougier 1972

continental rocks; Axe1rod 1960; Stephenson or Lower Tertiary

continental rocks Axelrod 1960; Carlquist 


\begin{tabular}{|c|c|c|c|}
\hline Island & $\frac{\text { Latitude } \&}{\text { Longitude }}$ & Age & Source \\
\hline Andamans-Ni cobars & $11.38 \mathrm{~N}, 92.17 \mathrm{E}$ & continental crust & Peter, Weeks \& Burns 1966 \\
\hline Andaman Island & $11.41 \mathrm{~N}, 92.46 \mathrm{E}$ & Eocene (?) & Glaessner 1943 \\
\hline Christmas & $10.35 \mathrm{~S}, 105.40 \mathrm{E}$ & $\begin{array}{l}\text { continental; or } \\
\text { Eocene }\end{array}$ & $\begin{array}{l}\text { Marshall 1927; Wilson 1963; } \\
\text { Van Balgooy } 1969\end{array}$ \\
\hline Wallaby Plateau & & continental crust & $\begin{array}{r}\text { Geomagnetism Correspondent } \\
1973\end{array}$ \\
\hline Exmouth P1ateau & & continental crust & $\begin{array}{r}\text { Geomagnetism Correspondent } \\
1973\end{array}$ \\
\hline Naturaliste Plateau & & continental crust & Petkovic 1975 \\
\hline Broken Ridge & & continental crust & Petkovic 1975 \\
\hline \multicolumn{4}{|l|}{ NDONESIA-PHILIPPINES } \\
\hline Langkawi Island & $6.22 \mathrm{~N}, 99.50 \mathrm{E}$ & Palaeozoic & Katili 1971 \\
\hline Riouw Archipe1ago & $0.49 \mathrm{~N}, 103.45 \mathrm{E}$ & Triassic & Umbgrove 1938 \\
\hline Sumatra & $2.06 \mathrm{~N}, 99.40 \mathrm{E}$ & continental rocks & $\begin{array}{l}\text { Woolnough 1903; Umbgrove } \\
1949 \text {; Carlquist } 1965\end{array}$ \\
\hline Java & $8.35 \mathrm{~S}, 111.11 \mathrm{E}$ & $\begin{array}{l}\text { continental } \\
\text { crust (?) }\end{array}$ & Woo1nough 1903; Cloud 1953 \\
\hline Bali & $8.00 \mathrm{~S}, 115.22 \mathrm{E}$ & continental & Carlquist 1965 \\
\hline Timor & $10.08 \mathrm{~S}, 125.00 \mathrm{E}$ & Permian & Umbgrove 1949 \\
\hline Letti Island & $8.12 \mathrm{~S}, 127.41 \mathrm{E}$ & Permian & Umbgrove 1949 \\
\hline Billiton Island & $2.50 \mathrm{~S}, 107.56 \mathrm{E}$ & $\begin{array}{l}\text { Triassic/ } \\
\text { Jurassic }\end{array}$ & $\begin{array}{l}\text { Harland, Smith \& Wilcock } \\
1964\end{array}$ \\
\hline $\begin{array}{l}\text { Barbar, Moa, Sawu, } \\
\text { Rotti }\end{array}$ & & Permian & Umbgrove 1949 \\
\hline Jamdena & $7.23 \mathrm{~S}, 130.30 \mathrm{E}$ & Jurassic & Umbgrove 1938 \\
\hline Bathurst Island & $11.35 \mathrm{~S}, 131.41 \mathrm{E}$ & Cretaceous & Wright 1963 \\
\hline $\begin{array}{l}\text { Tambelan \& Bunguran } \\
\text { Islands }\end{array}$ & $0.38 \mathrm{~N}, 107.38 \mathrm{E}$ & Late Cretaceous & Haile \& Bignell 1971 \\
\hline $\begin{array}{l}\text { Tanimbar, Boeton, } \\
\text { Boeroe, Ambon, Banka, } \\
\text { Billiton, Rendjoewa, } \\
\text { Savoe, Misool, the } \\
\text { Moluccas }\end{array}$ & & Triassic & 1938 \\
\hline
\end{tabular}


Oakley Shields

\begin{tabular}{|c|c|c|c|}
\hline Is 1and & $\frac{\text { Latitude } \varepsilon}{\text { Longitude }}$ & Age & Source \\
\hline Borneo & $0.25 \mathrm{~N}, 112.39 \mathrm{E}$ & $\begin{array}{l}\text { continenta1 } \\
\text { crust (?) }\end{array}$ & $\begin{array}{l}\text { Woolnough 1903; Reed 1949; } \\
\text { Cloud } 1953\end{array}$ \\
\hline Celebes & $2.15 \mathrm{~S}, 120.30 \mathrm{E}$ & Triassic & Umbgrove 1949 \\
\hline Soela Is 1ands & $2.20 S, 125.20 \mathrm{E}$ & $\begin{array}{c}\text { end of Palaeozoic } \\
\text { (?) }\end{array}$ & Umbgrove 1938 \\
\hline Kei Islands & $5.35 \mathrm{~S}, 132.45 \mathrm{E}$ & Eocene & G1aessner 1943 \\
\hline Ceram & $3.00 \mathrm{~S}, 129.00 \mathrm{E}$ & Triassic & Grunau 1965 \\
\hline Buru & $3.30 \mathrm{~S}, 126.30 \mathrm{E}$ & Jurassic & Arke11 1956 \\
\hline Obi & $1.25 S, 128.15 \mathrm{E}$ & Jurassic & Umbgrove 1949 \\
\hline Halmahera & $0.45 \mathrm{~N}, 128.45 \mathrm{E}$ & Cretaceous & Umbgrove 1938 \\
\hline Philippines & $14.25 \mathrm{~N}, 125.00 \mathrm{E}$ & $\begin{array}{l}\text { continental crust } \\
(?) \text {, in part }\end{array}$ & Cloud 1953; Carlquist 1965 \\
\hline Mindanao & $7.30 \mathrm{~N}, 125.10 \mathrm{E}$ & continental rocks & Irving 1952 \\
\hline Negros & $9.50 \mathrm{~N}, 121.45 \mathrm{E}$ & continental rocks & Irving 1952 \\
\hline Cebu & $10.22 \mathrm{~N}, 123.49 \mathrm{E}$ & Cretaceous (?) & Irving 1949 \\
\hline Palawan & $9.50 \mathrm{~N}, 117.38 \mathrm{E}$ & continental rocks & Irving 1952 \\
\hline Mindoro & $13.04 \mathrm{~N}, 121.06 \mathrm{E}$ & Jurassic (?) & Irving 1949; Arke11 1956 \\
\hline Luzon & $17.10 \mathrm{~N}, 119.45 \mathrm{E}$ & continental rocks & Irving 1952 \\
\hline \multicolumn{4}{|l|}{ RING SEA } \\
\hline St. Matthew Island & $60.25 \mathrm{~N}, 172.10 \mathrm{~W}$ & Upper Cretaceous & $\begin{array}{l}\text { Patton, Lanphere, Miller, } \\
\text { Scott } 1974\end{array}$ \\
\hline \multicolumn{4}{|l|}{ ACIFIC OCEAN } \\
\hline \multicolumn{4}{|l|}{ artem edge of Pacific } \\
\hline \multicolumn{4}{|l|}{ A1askan Peninsula: } \\
\hline A1eutian Islands & $52.40 \mathrm{~N}, 177.30 \mathrm{E}$ & $\begin{array}{l}\text { latest Cretaceous, } \\
\text { early Tertiary, } \\
\text { or Early 01igocene }\end{array}$ & $\begin{array}{l}\text { Burk 1965; Schol1, Marlow, } \\
\text { \& Buffington } 1975\end{array}$ \\
\hline $\begin{array}{l}\text { guyot in Aleutian } \\
\text { Trench }\end{array}$ & & $\begin{array}{l}\text { early Tertiary } \\
\text { or o1der }\end{array}$ & Wils on 1963 \\
\hline Adak Island & $51.40 \mathrm{~N}, 176.28 \mathrm{~W}$ & Permian & Packham \& Falvey 1971 \\
\hline $\begin{array}{l}\text { outer Shumagin } \\
\text { Islands }\end{array}$ & $55.22 \mathrm{~N}, 159.20 \mathrm{~W}$ & Upper Cretaceous & Burk 1965 \\
\hline
\end{tabular}




\begin{tabular}{llll}
\multicolumn{1}{c}{ Island } & $\frac{\text { Latitude \& }}{\text { Longitude }}$ & Age & Source \\
Sanak Island & $54.26 \mathrm{~N}, 162.40 \mathrm{~W}$ & Upper Cretaccous & Burk 1965 \\
$\begin{array}{l}\text { Semidi Is. \& } \\
\text { Chirikof Is. }\end{array}$ & $55.50 \mathrm{~N}, 155.35 \mathrm{~W}$ & Late Mesozoic & Burk 1965 \\
Kodiak Islands & $57.24 \mathrm{~N}, 153.32 \mathrm{~W}$ & Palaeozoic & Burk 1965
\end{tabular}

II. San Juan Is., Washington
San Juan Islands
$48.30,123.05 \mathrm{~W}$
Devonian
McLellan 1927

III Channel Is1ands

San Miguel I.

Santa Rosa

Santa Cruz I.

San Nicolas

Santa Catalina I.

$34.03 \mathrm{~N}, 120.23 \mathrm{~W}$

$34.02 \mathrm{~N}, 120.15 \mathrm{~W}$

$34.05 \mathrm{~N}, 119.55 \mathrm{~W}$

$33.14 \mathrm{~N}, 119.10 \mathrm{~W}$

$33.29 \mathrm{~N}, 118.37 \mathrm{~W}$

IV. Gulf of California

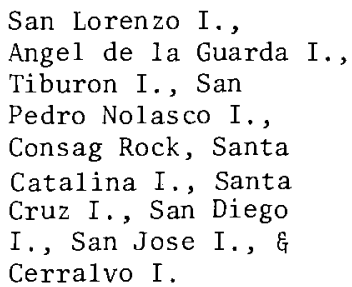

v. off the South American coast

$\begin{array}{lccc}\text { Galapagos Islands } & 0.10 \mathrm{~S}, 87.45 \mathrm{~W} & \text { continental biota } & \text { Baur } 1897 \\ \text { San Felix \& San } & 26.40 \mathrm{~S}, 80.00 \mathrm{~W} & \text { continental flora } & \text { Kuschel } 1963 \\ \text { Ambrosio } & & & \\ \text { Juan Fernandez } & 33.30 \mathrm{~S}, 79.00 \mathrm{~W} & \text { continental biota Kuschel } 1963\end{array}$

Northwest Pacific margin

$\begin{array}{llll}\begin{array}{l}\text { Kamchatka-Kuril- } \\ \text { Hokkaido Arc }\end{array} & \text { continental } & \text { Menard \& Ladd, } 1963 \\ \text { Sakhalin } & 51.52 \mathrm{~N}, 144.15 \mathrm{E} & \text { continental } & \text { Carlquist } 1965 \\ \text { Japan } & 36.30 \mathrm{~N}, 133.30 \mathrm{E} & \text { continental } & \text { Carlquist } 1965 \\ \text { Ryukyu Islands } & 26.00 \mathrm{~N}, 119.00 \mathrm{E} & \text { continental (?) } & \text { Cloud } 1953\end{array}$
pre-Tertiary Anderson 1950 basement Weaver 1969 Weaver 1969 Weaver 1969 Van Balgooy 1969 
Is 1 and

Taiwan

West Pacific

Bonin Islands

Southern Marianas

Saipan

Tinian

Rota

Guam

Mage 11 an Rise

Yap

Palau Islands

Babelthuap

Malakal

West Carolines

Truk

Maprong

Ponape

Map

Southwest Pacific

Ontong Java Plateau

(= Solomon Rise)

New Guinea

Bismarck Archipelago

New Britain

New Ireland
Latitude \&

$23.30 \mathrm{~N}, 122.20 \mathrm{E}$

continental

C1oud 1953; Carlquist 1965
$27.00 \mathrm{~N}, 142.10 \mathrm{E}$

$17.20 \mathrm{~N}, 145.00 \mathrm{E}$

$15.12 \mathrm{~N}, 145.43 \mathrm{E}$

Eocene

Midd1e Eocene

$14.58 \mathrm{~N}, 145.38 \mathrm{E} \quad$ Eocene

14.10N, 145.15E Eocene

$14.00 \mathrm{~N}, 143.20 \mathrm{E}$

Middle Eocene

Upper Jurassic

continental rocks

$7.15 \mathrm{~N}, 134.30 \mathrm{E}$

Middle Eocene

$7.30 \mathrm{~N}, 134.36 \mathrm{E}$

$7.20 \mathrm{~N}, 134.28 \mathrm{E}$

$9.30 \mathrm{~N}, 143.00 \mathrm{E}$

$7.28 \mathrm{~N}, 151.51 \mathrm{E}$

continental

continental

Eocene/01igocene

continenta 1 rocks

Daly 1916

$9.35 \mathrm{~N}, 138.11 \mathrm{E}$

Late Cretaceous

Hammond et al. 1975

$5.45 S, 140.00 \mathrm{E}$ continenta1 rocks Reed 1949; Umbgrove 1949.

$3.15 \mathrm{~S}, 150.45 \mathrm{E}$

$6.45 \mathrm{~S}, 149.38 \mathrm{E}$

3. $15 \mathrm{~S}, 152.30 \mathrm{E}$

C1oud 1953; Karing 1971; Karig et al., 1973; Karig \& Moore 1975

C1oud 1953

Hanzawa 1957; Co1e 1963; Tracey et al. 1964; Karig et al. 1973

Han zawa 1957

Han zawa 1957

Co1e 1963; Tracey et. al. 1964; Karig et. al.1973

Larson \& Chase 1972

Daly 1916; Gregory 1928, 1930

C1oud 1953; Hanzawa 1957; Karig et $\alpha$ l. 1973

Da1y 1916; Mason \& Corwin 1953; Hanzawa 1957

Marsha11 1911

Van Balgooy 1969

Daly 1916; Gregory 1928 (but see Bridge 1948)

Gregory 1928

Van Ba1gooy 1969

Cretaceous (?) Reed 1949

Lower Oligocene Reed, 1949 


\begin{tabular}{|c|c|c|c|}
\hline Is land & $\frac{\text { Latitude } \xi}{\text { Longitude }}$ & Age & Source \\
\hline Lou & $2.25 \mathrm{~S}, 147.23 \mathrm{E}$ & continental rocks & Daly 1916 \\
\hline Macada & & continenta1 rocks & Daly 1916 \\
\hline New Hanover & $2.37 \mathrm{~S}, 150.15 \mathrm{E}$ & continenta1 rocks & Daly 1916 \\
\hline New Mechlenburg & & continental rocks & Daly 1916 \\
\hline Solomon Islands & $7.00 \mathrm{~S}, 148.00 \mathrm{E}$ & continental & Baur 1897; Griffiths 1971 \\
\hline Guadalcana 1 & $9.48 \mathrm{~S}, 158.43 \mathrm{E}$ & continental rocks & Grover 1955 \\
\hline San Cristobal & $10.47 \mathrm{~S}, 162.17 \mathrm{E}$ & continental rocks & Reed 1949; Grover 1955 \\
\hline St. George & $8.30 S, 159.36 \mathrm{E}$ & continental rocks & Grover 1955 \\
\hline Vulavu & $8.31 \mathrm{~S}, 159.48 \mathrm{E}$ & continental rocks & Woolnough 1903 \\
\hline Faro (Fauro) & $6.55 \mathrm{~S}, 156.05 \mathrm{E}$ & continental rocks & Daly 1916 \\
\hline Shortland Is lands & $7.05 \mathrm{~S}, 155.45 \mathrm{E}$ & continental rocks & Daly 1916 \\
\hline Savo I. & $9.10 \mathrm{~S}, 159.50 \mathrm{E}$ & Lower Cretaceous & Coleman 1970 \\
\hline Russe11 I. & $9.16 \mathrm{~S}, 158.30 \mathrm{E}$ & Lower Cretaceous & Co1eman 1970 \\
\hline Choiseul & $7.30 \mathrm{~S}, 157.30 \mathrm{E}$ & $51.5 \mathrm{~m} . \mathrm{y}$. & Richards et al. 1966 \\
\hline Malaita & $8.38 \mathrm{~S}, 161.15 \mathrm{E}$ & Upper Cretaceous & Coleman 1966b, 1970 \\
\hline Malaita group & & $\begin{array}{l}\text { Oligocene or } \\
\text { older (?) }\end{array}$ & Univ. Sydney 1956 \\
\hline $\begin{array}{l}\text { Central Province - } \\
\text { (Bougainville, } \\
\text { Choiseul, Santa } \\
\text { Ysabel, F1orida } \\
\text { Group, Guada1cana1, } \\
\text { San Cristoval) }\end{array}$ & & $\begin{array}{l}\text { Mesozoic (probably } \\
\text { Cretaccous) basal } \\
\text { complex }\end{array}$ & Coleman 1966a \\
\hline San Jorge & $8.27 \mathrm{~S}, 159.35 \mathrm{E}$ & pre-Tertiary (?) & Univ. Sydney 1956 \\
\hline Florida I & $9.05 \mathrm{~S}, 160.15 \mathrm{E}$ & continental rocks & Marshal1 1911 \\
\hline Santa Ysabel & $8.00 S, 159.00 \mathrm{E}$ & pre-Tertiary (?) & Univ. Sydney 1956; Stanton \\
\hline Louisiade Ridge & & continental crust & Griffiths 1971 \\
\hline Renne11 Ridge & & continental crust & Griffiths 1971 \\
\hline Queensland P1ateau & & continenta1 crust & Griffiths 1971 \\
\hline
\end{tabular}


Oakley Shields

Island

New Hebrides
Malicolo
Spiritu Santo
Aneityum
Malekula
Maewo

New Caledonia

Fiji Archipelago

Vanua Levu

Viti Levu

Lau

Wallis Islands

Tonga Is lands

Eua I.

Kermadec Islands

Lord Howe Rise

Lord Howe Is 1 and

Norfolk Ridge

Norfolk Island

New Zea1and

Latitude G Longitude

16.02S, 169. 15E

16. $15 \mathrm{~S}, 167.30 \mathrm{E}$

$15.15 \mathrm{~S}, 166.50 \mathrm{E}$

$20.15 \mathrm{~S}, 169.49 \mathrm{E}$

$16.44 S, 167.45 \mathrm{E}$

$15.17 \mathrm{~S}, 168.16 \mathrm{E}$

$21.30 \mathrm{~S}, 165.30 \mathrm{E}$

$18.50 \mathrm{~S}, 175.00 \mathrm{E}$

$16.33 \mathrm{~S}, 179.15 \mathrm{E}$ or $17.28 \mathrm{~S}, 177.03 \mathrm{E}$

$18,00 \mathrm{~S}, 178.00 \mathrm{E}$

$18.20 \mathrm{~S}, 178.30 \mathrm{~W}$

13. $16 \mathrm{~S}, 176.15 \mathrm{~W}$

$18.50 \mathrm{~S}, 175.20 \mathrm{~W}$

$21.23 \mathrm{~S}, 174.55 \mathrm{~W}$

$30.30 \mathrm{~S}, 177.00 \mathrm{E}$ or Upper

$31.44 \mathrm{~S}, 157.56 \mathrm{E}$

\& flora

$29.05 \mathrm{~S}, 167.59 \mathrm{E}$

39. 14S, $169.30 \mathrm{E}$ continental rocks

(?) Axe1rod 1960

continental crust; Officer 1955; Griffiths 1971; Cretaceous

continental rocks

continental crust Griffiths 1971 continental rocks

Precambrian
Packham \& Falvey 1971;

McDougall \& Van Der Lingen 1974

\section{Source}

Reed 1949

Woolnough 1903

Woolnough 1903

Woolnough 1903

Coleman 1969, 1970

\& Tilton 1971;

Marshal1 1911; Avias 1953;

1969; Waterhouse 1971

oolnough 1903; Axe1rod 1960; Van Balgooy 1969

Marshall 1911; Reed 1949; tandard 1963; Van Balgooy 1969

Daly $1916 a$

Reed 1949; Aronson 1965; Aronson \& Tilton 1971 


\begin{tabular}{|c|c|c|c|}
\hline Island & $\frac{\text { Latitude } q}{\text { Longitude }}$ & Age & Source \\
\hline $\begin{array}{l}\text { Three Kings Islands } \\
\text { (N. New Zealand) }\end{array}$ & $34.09 \mathrm{~S}, 172.09 \mathrm{E}$ & Cretaceous & Bartrum 1948 \\
\hline Chatham Rise & & continental crust & Griffiths 1971 \\
\hline Chatham Islands & $44.00 \mathrm{~S}, 178.00 \mathrm{~W}$ & continental rocks & Axe1rod 1960; Carlquist 1965 \\
\hline Campbe11 P1ateau & & continental crust & Adams 1962; Griffiths 1971 \\
\hline Campbe11 Is land & $52.30 \mathrm{~S}, 169.00 \mathrm{E}$ & continental rocks & Axe1rod 1960; Carlquist 1965 \\
\hline Bounty Is 1 ands & $47.42 S, 179.05 E$ & $\begin{array}{l}\text { continental rocks; } \\
\text { or Triassic/ } \\
\text { Jurassic }\end{array}$ & $\begin{array}{l}\text { Axe1rod 1960; Wasserburg et } \\
\text { al. 1963; Carlquist } 1965\end{array}$ \\
\hline The Snares & $48.00 \mathrm{~S}, 116.30 \mathrm{E}$ & continental rocks & Daly 1916 . \\
\hline Auck 1and Is lands & $50.30 \mathrm{~S}, 166.30 \mathrm{E}$ & continental rocks & Daly 1916; Axelrod 1960 \\
\hline Disappointment & $50.35 \mathrm{~S}, 166.00 \mathrm{E}$ & continental rocks & Daly 1916 \\
\hline Macquarie Island & $54.36 \mathrm{~S}, 158.45 \mathrm{E}$ & continental rocks & Axelrod 1960; Carlquist 1965 \\
\hline Tasmania & $41.28 \mathrm{~S}, 142.30 \mathrm{E}$ & Precambrian & Reed 1949 \\
\hline Marie Byrd Land & $78.00 \mathrm{~S}, 140.00 \mathrm{~W}$ & Precambrian & Elliot 1975 \\
\hline Shatsky Rise & & Lower Cretaceous & $\begin{array}{l}\text { Larson \& Chase } 1972 ; \\
\text { Douglas \& Moullade } 1972\end{array}$ \\
\hline Koko Seamount & $34.47 \mathrm{~N}, 171.50 \mathrm{E}$ & $\begin{array}{l}\text { Eocene } \\
(46.4 \mathrm{~m} . \mathrm{y} .)\end{array}$ & C1ague \& Dalrymple 1973 \\
\hline Suiko Seamount & & $41 \mathrm{~m} . \mathrm{y}$. & $\begin{array}{l}\text { Jackson, Silver, \& Dalrymple } \\
1972\end{array}$ \\
\hline $\begin{array}{l}\text { Khatchaturian } \\
\text { Seamount }\end{array}$ & $28.08 \mathrm{~N}, 162.17 \mathrm{~W}$ & $65 \pm 3$ m.y. & Clague \& Dalrymple 1975 \\
\hline $\begin{array}{l}\text { Rachmaninoff } \\
\text { Seamount }\end{array}$ & $29.33 \mathrm{~N}, 163.22 \mathrm{~W}$ & $87 \pm 5 \mathrm{~m} \cdot \mathrm{y}$ & Clague \& Dalrymple 1975 \\
\hline Wentworth Seamount & $28.54 \mathrm{~N}, 177.52 \mathrm{~W}$ & $71 \pm 5 \mathrm{~m} \cdot \mathrm{y}$ & Clague \& Da1rymple 1975 \\
\hline Necker Island & $23.48 \mathrm{~S}, 164.25 \mathrm{~W}$ & $78 \pm 2$ m.y. & Clague \& Dalrymple 1975 \\
\hline $\begin{array}{l}3 \text { seamounts SW of } \\
\text { the Hawaiian Islands }\end{array}$ & & $\begin{array}{l}\text { Cretaceous } \\
(85-90 \text { m.y. })\end{array}$ & Dymond \& Windom 1968 \\
\hline Line Islands & $0.05 \mathrm{~N}, 157.00 \mathrm{~W}$ & $\begin{array}{l}\text { Upper } \\
\text { Cretaceous }\end{array}$ & Schlanger et al. 1974a, b \\
\hline
\end{tabular}


Islands $\frac{\text { Latitude } \xi}{\text { Longitude }}$

Horizon Guyot

Kapsitotwa Seamount

Guyot 19171

Hess Guyot

Cape Johnson Guyot

Shepard, Jacqueline, \& Menard seamounts or guyots

guyots in the northern Marshall area

Marshall Islands

Eniwetok

Bikini

Sylvania Guyot

Cook Is lands

Mauke

Mitiaro

Atiu

Aitutaki

Mangaia

Rarotonga

Northern Group

atolls

Manihiki Plateau

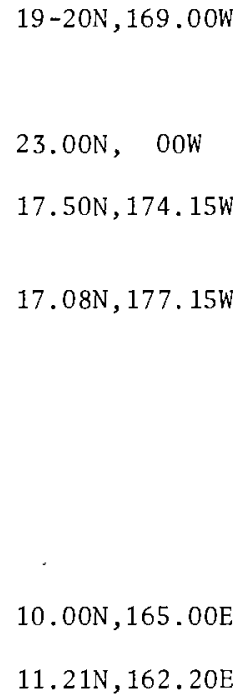

$10.00 \mathrm{~N}, 165.00 \mathrm{E}$

$11.21 \mathrm{~N}, 162.20 \mathrm{E}$

Eocene; or O1igocene (?)

Lower Eocene

$20.00 \mathrm{~S}, 158.00 \mathrm{~W}$

$20.09 S, 157.23 \mathrm{~W}$

$19.49 S, 157.43 W$

$20.00 \mathrm{~S}, 158.07 \mathrm{~W}$

$18.525,159.46 \mathrm{~W}$

$21.56 \mathrm{~S}, 157.56 \mathrm{~W}$

$20.405,163.00 \mathrm{~W}$

\section{Cretaceous \\ Early Tertiary}

Cretaceous Oligocene
Source

Hami 1ton 1956

Clague \& Dalrymple 1975

Hamilton 1956

Hamilton 1956

Hamilton 1956

Clague G Da1rymple 1975

Emery, Tracey, ६ Ladd 1954

Coleman 1969

(?) Cloud 1953; Hami1ton 1956; Cole 1963; Tracey et al. 1964

Emery, Tracey, \& Ladd 1954;

Hanzawa 1957; Cole 1963

Wilson 1963

Wood \& Hay 1970

Wood \& Hay 1970

Wilson 1963; Wood \& Hay 1970

Wilson 1963; Wood \& Hay 1970

end of Cretaceous; Marsha11 1927; Gregory 1930; or Eocene; or Wood 1967; Wood \& Hay 1970

Marsha11 1930

Wood \& Hay 1970

Sch1 anger et al. 1974b 


\begin{tabular}{|c|c|c|c|}
\hline Is 1and & $\frac{\text { Latitude } \varepsilon}{\text { Longitude }}$ & Age & Source \\
\hline Tubuai Islands & $23.23 \mathrm{~S}, 149.27 \mathrm{~W}$ & & \\
\hline Rimatara & $22.40 S, 152.45 W$ & continental rocks & $\begin{array}{l}\text { Wi11iams 1933; Wood \& Hay } \\
1970\end{array}$ \\
\hline Tubuai & $23.23 S, 149.27 \mathrm{~W}$ & $25 \pm 10 \mathrm{~m}, \mathrm{y}$. & Krummenacher \& Noetz 1 in 1967 \\
\hline Rapa & $27.36 \mathrm{~S}, 144.20 \mathrm{~W}$ & $\begin{array}{l}\text { continental rocks } \\
\text { \& flora }\end{array}$ & $\begin{array}{l}\text { Williams } 1933 \text {; Wilson } 1963 \\
\text { Van Balgooy } 1969 \text {; Woods \& } \\
\text { Hay } 1970\end{array}$ \\
\hline Society Islands & $17.00 \mathrm{~S}, 150.00 \mathrm{~W}$ & & \\
\hline Maupiti & $16.27 \mathrm{~S}, 152.15 \mathrm{~W}$ & $\begin{array}{r}\text { continental rocks } \\
(?)\end{array}$ & Gregory 1930 \\
\hline Bora-Bora & $16.30 \mathrm{~S}, 151.45 \mathrm{~W}$ & $\begin{array}{r}\text { continental rocks } \\
(?)\end{array}$ & Marsha11 1911; Da1y 1916 \\
\hline Moorea & $17.32 \mathrm{~S}, 149.50 \mathrm{~W}$ & $\begin{array}{r}\text { continental rocks } \\
(?)\end{array}$ & Wi1son 1963 \\
\hline Tahiti & $17.30 \mathrm{~S}, 149.30 \mathrm{~W}$ & $\begin{array}{l}\text { continental rocks; } \\
833 \mathrm{~m} \cdot \mathrm{y}, \text {; or } \\
156 \pm 15 \mathrm{~m} \cdot \mathrm{y} .\end{array}$ & $\begin{array}{l}\text { Wilson 1963; Krummenacher \& } \\
\text { Noetzlin 1967; Krummenacher } \\
\text { et al. 1972 }\end{array}$ \\
\hline Tahiti-Iti & $17.47 \mathrm{~S}, 149.14 \mathrm{~W}$ & continental rocks & Wi1son 1963 \\
\hline Tuamotu Ridge & & pre-early Eocene & Schlanger et az. $1974 \mathrm{a}$ \\
\hline Tuamotu Islands & $19.00 \mathrm{~S}, 142.00 \mathrm{~W}$ & & \\
\hline Makatea & $15.50 \mathrm{~S}, 148.15 \mathrm{~W}$ & Eocene & Wilson 1963 \\
\hline $\begin{array}{l}\text { guyot amid the } \\
\text { atolls of the } \\
\text { Tuamotu Archipelag }\end{array}$ & & Eocene & Menard \& Ladd 1963 \\
\hline $\begin{array}{l}\text { guyots among the } \\
\text { Tuamotu Islands }\end{array}$ & & $40-45 \mathrm{~m} \cdot \mathrm{y}$. & Vogt \& Ostenso 1967 \\
\hline Marquesas Islands & $8.50 \mathrm{~S}, 141.00 \mathrm{~W}$ & $\begin{array}{l}\text { continental } \\
\text { rocks (?) }\end{array}$ & Gregory 1930 \\
\hline Easter Is land & $26.50 \mathrm{~S}, 109.00 \mathrm{~W}$ & $\begin{array}{l}\text { continental } \\
\text { rocks (?) }\end{array}$ & $\begin{array}{l}\text { Marshal1 1911; Daly 1916; } \\
\text { Stearns 1945, p.617; King } \\
1962 \text { p.606, } 609\end{array}$ \\
\hline Sala-y-Gomez & $26.28 \mathrm{~S}, 105.28 \mathrm{~W}$ & continental rocks & King 1962, p. 606 \\
\hline
\end{tabular}


Oakley Shields

Island $\quad \frac{\text { Latitude \& }}{\underline{\text { Longitude }}} \quad$ Age $\quad$ Source

CARIBBEAN-- GULF OF MEXICO

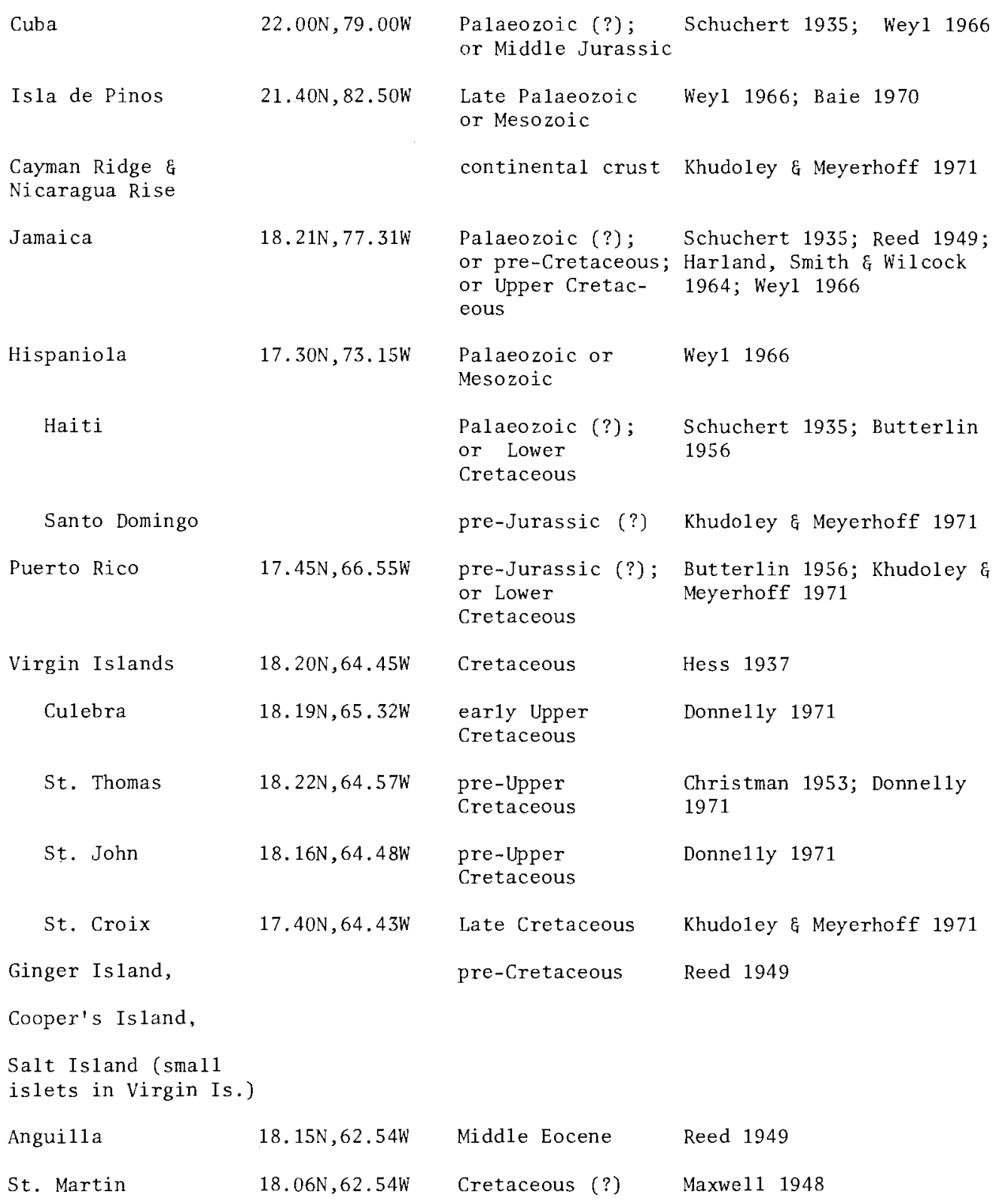




\begin{tabular}{|c|c|c|c|}
\hline Island & $\frac{\text { Latitude } \xi}{\text { Longitude }}$ & Age & Source \\
\hline St. Barthelemy & $17.55 \mathrm{~N}, 62.32 \mathrm{~W}$ & Middle Eocene (?) & $\begin{array}{l}\text { Christman 1953; Martin- } \\
\text { Kaye } 1969\end{array}$ \\
\hline $\begin{array}{l}\text { The "Limestone } \\
\text { Caribbees" (Sombrero, } \\
\text { Anguilla, St. Martin, } \\
\text { Tintamarre, St. } \\
\text { Barthelemy, Barbuda, } \\
\text { Antigua, Grande Terre } \\
\text { of Guadeloupe, } \\
\text { Desirade, \& Marie } \\
\text { Galante) }\end{array}$ & & Eocene or older & $\begin{array}{l}\text { Maxwel1 1948; Martin-Kaye } \\
1969\end{array}$ \\
\hline $\begin{array}{l}\text { Saba, St. Eustatius, } \\
\text { St. Kitts, } \\
\text { Gaudeloupe, Dominica, } \\
\text { Martinique, St. Lucia } \\
\text { St. Vincent, \& Grenad }\end{array}$ & & $\begin{array}{l}\text { ejected frag- } \\
\text { ments of } \\
\text { continental } \\
\text { basement }\end{array}$ & $\begin{array}{l}\text { Westermann \& Kiel 1961, } \\
\text { p.168 }\end{array}$ \\
\hline $\begin{array}{l}\text { "Volcanic Caribbees" } \\
\text { (from Saba in the } \\
\text { north to Grenada in } \\
\text { the south) }\end{array}$ & & Eocene & Wey1 1966 \\
\hline $\begin{array}{l}\text { Windward Is lands } \\
\text { (from Dominica to } \\
\text { Grenada) }\end{array}$ & & $\begin{array}{l}\text { basement of } \\
\text { trappean rocks } \\
\text { beneath early } \\
\text { Tertiary for- } \\
\text { mations }\end{array}$ & Spencer 1902 \\
\hline Antigua & $17.07 \mathrm{~N}, 61.32 \mathrm{~W}$ & pre-Cretaceous (?) & Reed 1949 \\
\hline Montserrat & $16.48 \mathrm{~N}, 62.00 \mathrm{~W}$ & $\begin{array}{l}\text { Late Cretaceous (?) } \\
\text { or Early Eocene }\end{array}$ & Reed 1949 \\
\hline Desirade & $16.21 \mathrm{~N}, 60.51 \mathrm{~W}$ & $\begin{array}{l}\text { Jurassic or } \\
\text { older }\end{array}$ & Meyerhoff 1974 \\
\hline Dominica & $15.24 \mathrm{~N}, 61.05 \mathrm{~W}$ & $\begin{array}{l}\text { pre-Tertiary (?); } \\
\text { or Eocene }\end{array}$ & Earle 1928; Reed 1949 \\
\hline Martinique & $14.30 \mathrm{~N}, 60.37 \mathrm{~W}$ & Cretaceous & Hil1 1905; Hess 1937 \\
\hline Bequia & $13.00 \mathrm{~N}, 61.08 \mathrm{~W}$ & Eocene (?) & Martin-Kaye 1969 \\
\hline Mustique & $12.53 \mathrm{~N}, 61.03 \mathrm{~W}$ & Lower Eocene (?) & Martin-Kaye 1969 \\
\hline Canouan & $12.44 \mathrm{~N}, 61.10 \mathrm{~W}$ & Upper Eocene (?) & Martin-Kaye 1969 \\
\hline Mayreau & $12.39 \mathrm{~N}, 61.23 \mathrm{~W}$ & Lower Eocene (?) & Martin-Kaye 1969 \\
\hline Carriacou & $12.28 \mathrm{~N}, 61.20 \mathrm{~W}$ & Upper Eocene & Martin-Kaye 1969 \\
\hline Petit Martinique & $12.30 \mathrm{~N}, 61.25 \mathrm{~W}$ & Upper Eocene & Martin-Kaye 1969 \\
\hline
\end{tabular}


Oakley Shields

\begin{tabular}{|c|c|c|c|}
\hline Is 1 and & $\frac{\text { Latitude } \varepsilon}{\text { Longitude }}$ & Age & Source \\
\hline Grenada & $12.02 \mathrm{~N}, 61.27 \mathrm{~W}$ & Upper Eocene & Martin-Kaye 1969 \\
\hline Barbados & $13.30 \mathrm{~N}, 59.48 \mathrm{~W}$ & Early Eocene & $\begin{array}{l}\text { Maxwe11 1948; Christman 1953; } \\
\text { Wey1 } 1966\end{array}$ \\
\hline Tobago & $11.15 \mathrm{~N}, 60.30 \mathrm{~W}$ & Cretaceous & $\begin{array}{l}\text { Christman 1953; Butter1 in } \\
1956\end{array}$ \\
\hline Trinidad & $10.30 \mathrm{~N}, 60.30 \mathrm{~W}$ & Jurassic & $\begin{array}{l}\text { Christman 1953; Butterlin } \\
1956\end{array}$ \\
\hline Patos Is 1 and & $10.36 \mathrm{~N}, 61.54 \mathrm{~W}$ & Early Cretaceous & Kugler 1974 \\
\hline Margarita & $11.00 \mathrm{~N}, 64.15 \mathrm{~W}$ & $\begin{array}{l}\text { Mesozoic; or } \\
\text { Cretaceous (?) }\end{array}$ & Butterlin 1956; Weyl 1966 \\
\hline Bonaire & $12.10 \mathrm{~N}, 68.15 \mathrm{~W}$ & Upper Cretaceous & Maxwe11 1948; Butterlin 1956 \\
\hline Curacao & $12.12 \mathrm{~N}, 68.58 \mathrm{~W}$ & Upper Cretaceous & Maxwell 1948; Butterlin 1956 \\
\hline Aruba & $12.29 \mathrm{~N}, 70.00 \mathrm{~W}$ & Upper Cretaceous & Maxwe11 1948; Butterlin 1956 \\
\hline Gran Roque & $11.50 \mathrm{~N}, 66.45 \mathrm{~W}$ & $\begin{array}{r}\text { Upper Cretaceous } \\
(?)\end{array}$ & Butterlin 1956 \\
\hline Los Hermanos & $11.45 \mathrm{~N}, 66.28 \mathrm{~W}$ & $\begin{array}{r}\text { Upper Cretaceous } \\
\text { (?) }\end{array}$ & Butterlin 1956 \\
\hline Blanquilla & $11.51 \mathrm{~N} 64.37 \mathrm{~W}$ & early Eocene & Butterlin 1956 \\
\hline Los Testigos & $11.22 \mathrm{~N} .63 .06 \mathrm{~W}$ & early Eocene & Butter1in 1956 \\
\hline
\end{tabular}

DISCUSSION

It is illogical to assume that the oldest ages determined for islands are all correct, as more recent volcanic outpourings have been obscured older, underlying plutonic and metamorphic basement rocks. Only more geologic exploration, dredging, and drilling operations will uncover the answers. Examples follow of islands where only comparatively recent ages have been determined that instead may be continental in origin. McBirney and Williams (1969) reported fossils of late Miocene age for the Galapagos Islands; Cox and Dalrymple (1966) found that K-Ar ages from whole-rock basalt samples for six of the Galapagos islands ranged from only .09 to $4.2 \mathrm{~m} . y .01 \mathrm{~d}$. Yet Baur (1897) showed the biota to be decidedly continental. Also, giant land-tortoises occur in the Galapagos, each species restricted to a single island (Lucas 1891). Mauritius and Rodriguez are thought to be recent volcanoes of 1.6 and 8 m.y. respective$1 y$, by radiometric methods (McDouga11, Upton, \& Wadsworth 1965; McDouga11 and Chamalaum 1969). However, they possess giant land-tortoises (Lucas 1891), large flightless ground-birds, and flightless rails (Wallace 1880), evidence for a past continental contact or derivation. The last example is the Tristan da Cunha group for which Baker, Gass, Harris, and Le Maitre (1964, p. 586) reported radiometric ages (K-Ar) of only $6 \pm 1.5 \mathrm{~m} . y$. for Inaccessible Island and $4 \mathrm{~m} . y$. for Gough Island. But an obvious archaic relict, a highly-modified flightless rail (Atzantisia rogersi), is restricted to Inaccessible, and an endemic 1 and rail genus (Porphyriomis) occurs on Gough (Rand 1955). Thus both islands need a direct continental contact. in the past to explain 
this distribution. Future drilling on supposedly young volcanic islands, such as the Hawaiian Islands (McDougal1 1964) and Samoan Islands (Stearns 1944) of Pliocene age, may disclose the presence of much older rocks.

Crust of continental thickness is found beneath some islands, as determined seismically and by gravity anomalies. Over much of New Zealand the crust has a typical continental thickness of 30-40 km (Thomson \& Evison 1962). The Bouguer anomaly map for Viti Levu, Fiji, indicates a crust $30 \mathrm{~km}$ thick (Lillie and Brothers 1970). Lord Howe Rise is $29 \mathrm{~km}$ thick and thus is probably continental in origin (Shor, Kirk, and Menard 1971). Norfolk Ridge (22 km thick) and Campbell Plateau (average $20 \mathrm{~km}$ thick) are thought to probably be of continental origin (Shor, Kirk and Menard 1971; Adams 1962). Gran Canaria (15 km thick) in the Canary Islands "lies in the transition zone between oceanic and continental crust" (Bosshard and Macfarlane 1970). However, a probable high heat flow below Cyprus, the Shatsky Rise, and the Ontong Java plateau has resulted in the up-buckling of the seafloor and a lowering of the Moho discontinuity, a process probably also taking place on Malaita in the Solomons, Macquarie Island, and elsewhere (Carey pers. comm.). Similarly the Agulhas Plateau, $18 \mathrm{~km}$ thick, is underlain by arched and faulted oceanic basement (Scrutton 1973; Emery et al. 1975, p.32). Thus one cannot judge continental vs. non-continental on seismic crustal thickness alone.

Clague and Dalrymple (1975) recently noted that volcanic rocks from two seamounts in the Musicians Seamounts and Necker Island, and Wentworth Seamount on the Hawaiian Ridge, showed ages at or close to their surrounding Cretaceous seafloor, indicating they formed at the crest of the East Pacific Rise. Similarly three cores from the seafloor in the Tuamotu Islands yield Eocene ages (Riede1 \& Funnel1 1964), the same as guyots and Makatea of the Tuamotu Islands. Also, the Line Islands were $79-85 \mathrm{~m} . \mathrm{y}$. old when flow volcanism ceased on a $1270 \mathrm{~km}$ long segment of the chain (Schlanger $1974 \mathrm{a}, \mathrm{b})$ and may have been in contact with the South American continent at that time, since on Palmyra Atoll there is an endemic species of land-leech, an organism unlikely to be bird- or oversea-dispersed (Richardson 1975, and in $2 i t t$. ).

It is instructive to compare the oldest ages of islands with the seafloor ages of the oceans and seas containing them. In each case some of the islands predate the opening of the ocean. Thus the western Mediterranean opened in the Upper Miocene (Vogt, Higgs, \& Johnson 1971), while many of the islands there are Upper Palaeozoic in age. The Scotia Sea formed in the Lower Miocene (Barker 1970), but the islands are Jurassic to Precambrian in age. The Gulf of Mexico probably formed in the Lower Cretaceous (the age of the Straits of Florida given by Wilhelm \& Ewing 1972) and the Caribbean has five sites of Upper Cretaceous age from DSDP cores (Edgar et al. 1971), yet most of the Greater Antilles date back to the Palaeozoic. Much of the southwest Pacific formed in Lower to Upper Cretaceous times (Schlanger et al.1974b; Kennett et al. 1973; Hayes \& Ringis 1973), while continental rocks and crust are reported for many of the islands and plateaus. There is no seafloor older than the Upper Jurassic reported for the Atlantic, Indian, and Pacific Oceans (Pitman, Larson \& Herron 1974; Larson 1975), yet in all three at least some of the islands and plateaus are older. The above suggests that some continental splinter fragments have been left as debris, plucked off the margins of rifting continents as ocean basins formed by seafloor spreading. In the Pacific, the distribution pattern of continental islands does not always conform to the andesite-1ine boundary of earlier authors. 


\section{ACKNOWLEDGEMENTS}

I thank Mr. A. Bush and Professor S. Warren Carey of the University of Tasmania for helpful discussions. This work was supported by an NSF Graduate Traineeship while at University of California, Davis, and an ARGC grant to Professor S. Warren Carey.

\section{REFERENCES}

Adams, R.D., 1962: Thickness of the earth's crush beneath the Campbell Plateau:N.Z.J. Geoz. Geophys., 5, 74-85.

Anderson, C.A., 1950: 1940 E.W. Scripps Cruise to the Gulf of California. Part I. Geology of islands and neighbouring land areas: Geol. Soc. Am. Mem. 43, 1-53

Arke11, W.J., 1956: JURASSIC GEOLOGY OF THE WORLD. Oliver \& Boyd, Edinburgh 806 pp.

Aronson, J.L., 1965: Recomnaissance rubidium-strontium geochronology of New Zealand plutonic and metamorphic rock. N.Z.J. Geol. Geophys., $\underline{8}, 401-423$.

Aronson, J.L., and Tilton, G.R., 1971: Probable Precambrian detrital zircons in New Caledonia and Southwest Pacific continental structure.Geol. Soc. Am. BuZZ., 82 3449-3456.

Avias, J., 1953: Contribution a l'etude stratigraphique et paleontologique de 1a Nouvelle-Caledonie centrale. Sci. Terre, 1, 1-276.

Axe1rod, D.I., 1960, The evolution of flowering plants, in Tax, S., THE EVOLUTION OF LIFE, vol. 1 : The University of Chicago Press, Chicago. 227-305.

Baie, L.F., 1970: Possible structural link between Yucatan and Cuba. Bulz., Am. Assoc. Pet. Geol. 54, 2204-2207.

Baker, B.H., and Miller, J.A., 1963: Geology and geochronology of the Seychelles Islands and structure of the floor of the Arabian Sea: Nature, 199, 346-348.

Baker, P.E., Gass, I.G., Harris, P.G., and Le Maitre, R.W., 1964: The volcanological report of the Royal Society Expedition to Tristan da Cunha, 1962: Phizos. Trans. Soc. London A256, 439-578.

Barker, P.F., 1970: P1ate tectonics of the Scotia Sea region: Nature, 228, 1293-1296.

Bartrum, J.A., 1948: Report on rocks collected by Mr. G.A. Buddle from islands of the Three Kings Group. Rec. Auckzand Inst. Mus. 3, 205-206.

Baur, G., 1897: New observations on the origin of the Galapagos Islands, with remarks on the geological age of the Pacific Ocean. Am. Nat., 31, 661-680.

Black, M., Hil1, M.N., Laughton, A.S., and Matthews, D.H., 1964: Three non-magnetic seamounts off the Iberian coast. Q. J. Geol. Soc. London. 120, 477-517.

Bosshard, E., and Macfarlane, D.J., 1970: Crustal structure of the western Canary Islands from seismic refraction and gravity data. J.Geophys. Res. 75, 4901-4918.

Bridge, J., 1948: A restudy of the reported occurrence of schist on Truk, Eastern Caroline Islands, Pacific Sei. , 2, 216-222. 
Burk, C.A., 1965: Geology of the Alaska Peninsula-island arc and continental margin (part I). Geol. Soc. Am. Mem. 99, p. 1-250.

Butterlin, J., 1956: LA CONSTITUTION GEOLOGIQUE ET LA STRUCTURE DES ANTILLES. Centre National de la Reserche Scientifique, $453 \mathrm{pp}$.

Carlquist, S., 1965: ISLAND LIFE: A NATURAL HISTORY OF THE ISLANDS OF THE WORLD: The Natural History Press, Garden City. 451 pp.

Chernysheva, V.I., 1971: Greenstone-altered rocks of rift zones in median ridges of Indian Ocean. Int. Geol. Rev, , 13, 903-913.

Christman, R.A., 1953: Geology of St. Bartholomew, St. Martin, and Anguilla, Lesser Antilles. Geol. Soc. Am. Bulz., 64, 65-96.

Clague, D.A., and Dalrymple, G.B., 1973: Age of Koko Seamount, Emperor Seamount Chain. Earth Planet Sci. Lett. 17, 411-415.

, 1975: Cretaceous K-Ar ages of volcanic rocks from the Musicians Seamounts and the Hawaiian Ridge. Geophys. Res. Letters. 2, 305-308.

Cloud, P.E., 1953 (1956) : Provisional correlation of selected Cenozoic sequences in the western and central Pacific. 8th Pac. Sci. Congr. Proc., 2, 555-574.

Cole, W.S., 1960: Upper Eocene and 01igocene 1arger foraminifera from Viti Levu, Fiji. U.S. Geol. Surv. Prof. Pap. 374-A, A1-A7, 3 pls.

, 1963: Tertiary larger foraminifera from Guam. U.S. Geol. Surv. Prof. Pap. 403-E, E1-E28, $11 \mathrm{p} 1 \mathrm{~s}$.

Coleman, P.J., 1966a: The Solomon Islands as an island arc. Nature, 211, 1249-1251.

, 1966b: Upper Cretaceous (Senonian) bathyal pelagic sediments with Globotruncana from the Solomon Islands J. Geol. Soc. Aust. 13, p. 439-447.

, 1969: Derived Eocene larger foraminifera on Maewo, eastern New Hebrides, and their South-West Pacific implications. New Hebrides Geol. Surv. Rep. $1967,2 \mathrm{pp}$.

1970: Geology of the Solomon and New Hebrides Islands, as part of the Melanesian re-entrant, southwest Pacific. Pac. Sci., 24, 289-314.

Cormier, R.F., 1973: Radiometric ages of granitic rocks, Cape Breton Islands: discussion. Can. J. Earth Sci., 10, 1470 .

Cox A., and Da1rymple, G.B., 1966: Palaeomagnetism and potassium-argon ages of some volcanic rocks from the Galapagos Islands. Nature, 209, 776-777.

Crawford, A.R., and 0liver, R.L., 1969: The Precambrian geochronology of Ceylon. spec. Geol. Soc. Aust. Publ. 2, 283-306.

Daly, R.A., 1916a: Petrography of the Pacific Islands. Geol. Soc. Am. BuZl., 27, 325-344.

, 1916b: Problems of the Pacific Islands. Am. J. Sci., 191, 153-186. 
Dalzie1, I.W.D., 1972: K-Ar dating of rocks from Elephant Is1and, South Scotia Ridge. Geol. Soc. Am. BuZz., 83, 1887-1894.

Dietz, R.S., and Sprol1, W.P., 1970: East Canary Islands as a microcontinent within the Africa-North America continental drift fit. Nature, 226, 1043-1045.

Donne11y, T.W., ed., 1971: Caribbean geophysical, tectonic, and petrologic studies. Geol. Soc. Am. Mem. 130, 1-224.

Douglas, R.G., and Moullade, M., 1972: Age of the basal sediments on the Shatsky Rise, western North Pacific Ocean. Geol. Soc. Am. Bulz., 83, 1163-1168.

Dymond, J., and Windom, H.L., 1968: Cretaceous K-Ar ages from Pacific Ocean seamounts. Earth Planet. Soi. Lett. 4, 47-52.

Earle, K.W., 1928: Geological notes on the Island of Dominica, B.W.I. Geot. Mag., $65,169-187$.

Edgar, N.T., et al., 1971: Deep Sea Drilling Project.Geotimes, 16 (4), 12-16.

El1iott, D.H., 1975: Tectonics of Antarctica: a review. Am. J. Sei. 275A, 45-306.

Emery, K.O., Tracey, J.I., Jr., and Ladd, H.S., 1954: Geology of Bikini and nearby ato11s. Part 1, geology. U.S. Geol. Surv. Prof. Pap. 260-A, 1-265.

, Uchupi, E., Bowin, C.O., Phillips, J., and Simpson, E.S.W., 1975:

Continental margin off western Africa: Cape St. Francis (South Africa) to Walvis Ridge (south-west Africa): Bull. Am. Assoc. Pet.Geol. 59, 3-59.

Enge1en, G.B., 1964: A hypothesis on the origin of the Bermuda Rise. Tectonophysics, $1,85-93$.

Flower, M.F.J., and Strong, D.F., 1969: The significance of sandstone inclusions in lavas of the Comores Archipelago. Earth Planet. Soi. Lett. 7, 47-50.

Forbes, H.O., ed., 1903: THE NATURAL HISTORY OF SOKOTRA AND ADB-EL-KURI, FORMING A MONOGRAPH OF THE ISLANDS. R.H. Porter, London. $598 \mathrm{pp}$.

Fox, P.J., Heezen, B.C., and Johnson, G.L., 1970: Jurassic sandstone from the tropical Atlantic. Science, 170, 1402-1404.

Geomagnetism Correspondent, 1973: Age of the Indian Ocean. Nature London Phys. Sci., $243,105$.

Glaessner, M.F., 1943: Problems of stratigraphic correlation in the Indo-Pacific region. Proc. R. Soc. Viat. n.s., 55, 41-80.

Gregory, H.E., 1928 (1926): Types of Pacific islands. 3rd Pac. Sci. Congr. Proc., 2, 1663-1673.

Gregory, J.W., 1930: The geological history of the Pacific Ocean. Q. J. Geol. Soc. London, 86, 1xxii-cxxxvi.

Griffiths, J.R., 1971: Reconstruction of the southwest Pacific margin of Gondwana1and. Nature, 234, 203-207.

Grover, J.C., 1955: Geology, mineral deposits and prospects of mining development in the British Solomon Islands Protectorate. Geol. Survey British Solomon Isls. Mem. Interim, 1, 1-108. 
Grunau, H.R., 1965: Radiolarian cherts and associated rocks in space and time. Eclogae, Geoz. Helv., 58, 157-208.

de Haga Haig, H., 1895: The physical features and geology of Mauritius. Q. J. Geol. Soc. London. $51,463-471$.

Haile, N.S., and Bignel1, J.D., 1971: Late Cretaceous age based on K/Ar dates of granitic rock from the Tambelan and Bunguran Islands, Sunda Shelf, Indonesia. Geol. Mijnb. 50, 687-690.

Hamilton, E.L., 1956: Sunken islands of the mid-Pacific mountains. Geol. Soc. Am. Mem. 64, 1-97.

Hammond, S.R., Kroenke, L.W., Theyer, F., and Keeling, D.L. 1975: Late Cretaceous and Palaeogene palaeolatitudes of the Ontong Java Plateau. Nature, 255, 46-47.

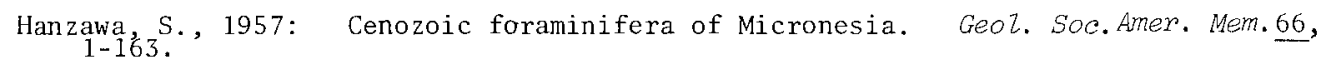
Harland, W.B., Smith, A.G., and Wilcock, B., eds., 1964: THE PHANEROZOIC TIME-SCALE. Geologica1 Society of London, $458 \mathrm{pp}$.

Hayes, D.E., and Ringis, J., 1973: The early opening of the Central Tasman Sea in Fraser, R., Oceanography of the South Pacific 1972. New Zealand National Commission for UNESCO, Wellington, pp.338

Hess, H.H., 1937: Geological interpretation of data collected on cruise of U.S.S. Barracuda in the West Indies - preliminary report. Trans. Am. Geophys. Union., $18,69-77$.

Hi11, R.T., 1905: Pele and the evolution of the Windward Archipelago. Geo. Soc. Am. Buzz., 16, 243-288.

Irving, E.M., 1949: Problems in the basement complex geology of Philippines. 7 th Pac. Sei. Congr. Proc., 2, 22.

, 1952: Geological history and petroleum possibilities of the Philippines. Bull. Am. Assoc. Pet. Geoz. 36, 437-476 (see fig. 4).

Jackson, E.D., Silver, E.A., and Dalrymple, G.B., 1972: Hawaiian-Emperor chain and its relation to Cenozoic circumpacific tectonics. Geol. Soc. Am. Buzz, , 83, 601-618.

Karig, D.E., 1971: Structural history of the Mariana Island arc system. Geol. Soc. Am. Buzz., 82, 323-344.

and Moore, G.F., 1975: Tectonic complexities in the Bonin Arc system.

Tectonophysies, $27,97-118$. $45 \overline{8-461}$.

and 12 others, 1973: Origin of the West Philippine Basin. Nature, 246,

Katili, J.A., 1971: A review of the geotectonic theories and tectonic maps of Indonesia. Earth-Sei. Rev. 7, 143-163.

Katz, M.B., 1971: The Precambrian metamorphic rocks of Ceylon. Geoz. Rundschau. $60, \quad 1523-1549$. 
Oakley Shields

Kennett, J.P., et al., 1973: Deep-sea drilling in the roaring 40s. Geotimes, 18 (7), $14-17$.

Khudoley, K.M., and Meyerhoff, A.A. 1971: Paleogeography and geologic history of Greater Antilles. Geol. Soc. Am. Mem. 129, 1-199.

King, L.C., 1962: THE MORPHOLOGY OF THE EARTH. Hafner Pub1. Co., New York, 699 pp.

Klemme, H.D., 1958: Regional geology of circum-Mediterranean region: Buzz. Am.Assoc. Pet. Geol., 42, 477-512.

Krummenacher, D., and Noetzlin, J., 1967: Ages isotopiques K/A de roches prelevies dans les possessions francaises du Pacifique Soc. geol. Fronce Bulz., Ser. 7. 8, $173-175$.

, et al., 1972: Potassium-argon ages from xenoliths and differentiatin coarse-grained rocks from the center of the island of Tahiti, French Polynesia (abs.). Geol. Soc. Am. Abs. with Programs, 4, 186.

Kugler, H.G., 1974: The geology of Patos Island (East Venezuela). Eclogae. Geol. Hezv., 67, 469-478.

Kuschel, G., 1963: Composition and relationship of the terrestrial faunas of Easter, Juan Fernandez, Desventuradas, and Galapagos Islands. Occ. Pap. Calif. Acad. Sci., 44, 79-95.

Larson, R.L., 1975: Late Jurassic sea-floor spreading in the eastern Indian Ocean. Geology, 3, 69-71.

, and Chase, C.G., 1972: Late Mesozoic evolution of the western Pacific Ocean. Geol. Soc. Am. Buzl., 83, 3627-3644.

Lillie, A.R., and Brothers, R.N., 1970: The geology of New Caledonia. N.Z.J. Geol. Geophys., 13, 145-183.

Lister, J.J., 1891: Notes on the geology of the Tonga Islands. Q.J. Geoz. Soc. Lond., $47,590-617$.

Lucas, F.A., 1891: The Galapagos and Mascarene tortoises. Smithson. Inst. Rep. 1889, 643-647.

Lynts, G.W., 1970: Conceptual model of the Bahamian Platform for the last 135 million years. Nature, 225, 1226-1228.

McBirney, A.R., and Williams, H., 1969: Geology and petrology of the Galapagos Islands. Geol. Soc. Am. Mem. 118, 1-197.

McDouga11, I., 1964: Potassium-argon ages from lavas of the Hawaiian Islands. Geoz. Soc. Am. Buzt., 75, 107-128.

, and Chamalaun, F.H., 1969: Isotopic dating and geomagnetic polarity studies on volcanic rocks from Mauritius, Indian Ocean. Geol. Soc. Bull., 80 , $1419-1442$.

Upton, B.G.J., and Wadsworth, W.J. 1965: A geological reconnaissance of Rodriguez Island, Indian Ocean. Nature, 206, 26.27.

, and Van Der Lingen, G.J., 1974: Age of rhyolites of the Lord Howe Rise and the evolution of the southwest Pacific Ocean. Earth Planet. Sci. Lett. 21 $117-126$. 
Mclellan, R.D., 1927: The geology of the San Juan Islands. Univ. Wash. Publ. Geol. 2, $1-185$.

Marshal1, P., 1911: Oceania. Hondb. Reg. Geol., 9, 1-36. , 1927: Geology of Mangaia. Bull. Bemice P Bishop Mus. 36, 1-48. 1930: Geology of Rarotonga and Atiu. Bul2. Bemice P Bishop Mus., 72 , $1-75$.

Martin-Kaye, P.H.A., 1969: A summary of the geology of the Lesser Antilles. Overseas Geol. Miner. Resour. 10, 172-206.

Mason, A.C., and Corwin, G., 1953: Geology of the Palau Islands. Geol. Soc. Am. BuZ2., 64, 1451.

Matthews, D.W., and Davies, D., 1966: Geophysical studies of the Seychelles Bank. Phizos. Trans. Roy. Soc. London A 259, 227-239.

Maxwell, J.C., 1948: Geology of Tobago, British West Indies. Geol. Soc. Am. Bull., 59, $801-854$.

Menard, H.W., and Ladd, H.S., 1963: Oceanic islands, seamounts, guyots and atol1s, in Hill, M.N., THE SEA, vol.3. Interscience Publishers, New York. pp. 365-387.

Meyerhoff, A.A., 1974: The Caribbean "plate". Geol. Soc. Am. Abs. with Programs, 6. 869 .

Mitche11-Thome, R.C., 1970: Geology of the South Atlantic Islands. Beitr. Reg. Ceol. Erde, $10,1-367$. 63, $1179-1216$.

1974: The sedimentary rocks of Macaronesia. Geol. Rundsch,

Nougier, J., 1972: Geochronology of the volcanic activity in Iles Kerguelen, in Adie, R.J., ANTARCTIC GEOLOGY AND GEOPHYSICS. Universitetsforlaget, Os1o. pp. 803-808.

Officer, C.B., 1955: Southwest Pacific crustal structure. Am. Geophys. Union Trans., 36, 449-459.

Packham, G.H., and Falvey, D.A., 1971: An hypothesis for the formation of marginal seas in the western Pacific.Tectonophysies, 11, 79-109.

Patton, W.W., Jr., Lanphere, M.A., Miller, T.P., and Scott, R.A. 1974: Age and tectonic significance of volcanic rocks on St. Matthew Island, Bering Sea, Alaska. Geo2. Soc. Am. Abs. with Programs, 6, 905-906.

Perfit, M., Heezen, B.C., and Rawson, M., 1974: Metamorphic rocks from the Puerto Rico Trench. Geol. Soe. Am. Abs. with Progroms, 6, 907-908.

Peter, G., Weeks, L.A., and Burns, R.E., 1966: A reconnaissance geophysica1 survey in the Andaman Sea and across the Andaman-Nicobar Island arc. J. Geophys. Res. $71, \quad 495-509$.

Petkovic, P., 1975: Origin of the Naturaliste Plateau. Nature, 253, 30-33. 
Pidgeon, R.T., and Hopgood, A.M. 1975: Geochronology of Archaean gneisses and tonalites from north of the Frederikshabs isblink, S.W. Greenland, Geochim. Cosmochim. Acta, 39, 1333-1346.

Pitman, W.C., III, Larson, R.L., and Herron, E.M., 1974: The age of the ocean basins. Geoz. Soc. Am. 2 charts.

Rand, A.L., 1955: The origin of the land birds of Tristan da Cunha. Fiezdiana, Zool. $37, \quad 139-166$.

Reed, F.R.C., 1949: THE GEOLOGY OF THE BRITISH EMPIRE, 2nd ed. Edward Arnold and Co., London $764 \mathrm{pp}$.

Reyno1ds, P.H., 1973: Deep Dri11-1972: geochronology of the Bermuda dri11 core, Am. Geophys. Union Trans, 54, 485.

Richards, J.R., Cooper, J.A., Webb, A.W., and Coleman, P.J., 1966: Potassium-argon measurements of the age of basal schists in the British Solomon Islands. Nature, 211, 1251-1252.

Richardson, L.R., 1975: A contribution to the general zoology of the land-leeches (Hirudinea: Haemadipsoidea superfom. nov.) Acta Zooz. Hung., 21, $119-152$.

Riede1, W.R., and Funne11, B.M., 1964: Tertiary sediment cores and microfossils from the Pacific Ocean floor. Q. J. Geol. Soc. London. 120, 305-368.

Roberts, D.G., 1975: Sediment distribution on the Rockall Bank, Rockall Plateau. Mar. Geol., 19, 239-257.

, Ardus, D.A., and Dearnley, R., 1973: Precambrian rocks drilled on the Bank. Nature (London) Phys. Sci., 244, 21-23.

Rothe, P., 1974: Canary Islands - origin and evolution. Naturwissenschaften, 61, $526-533$.

Schlanger, S.O., and 10 others, 1974a: Linear island chain chronologies: Leg 33 Deep Sea Drilling results from the Line and Tuamotu Islands. Geol. Soc. Am. Abs. with Programs, 6, 941.

, 1974b: Leg 33, Deep Sea Drilling Project, testing a hot-spot theory. Geotimes, 19 (3), 16-20.

Scholl, D.W., Marlow, M.S., and Buffington, E.C., 1975: Sumit basins of Aleutian Ridge, North Pacific. Buzz. Am. Assoc. Pet. Geol. 59, 799-816.

Schuchert, C., 1935: HISTORICAL GEOLOGY OF THE ANTILLEAN-CARIBBEAN REGION. John Wiley and Sons, New York, $811 \mathrm{pp}$.

Scrutton, R.A., 1973: Structure and evolution of the sea floor south of South Africa. Earth Planet. Sci. Lett., 19, 250-256.

Shor, G.G., Jr., Kirk, H.K., and Menard, H.W., 1971: Crustal structure of the Melanesian area. J. Geophys. Res. 76, 2562-2586.

Simpson, E.S.W., 1950: The geology and mineral resources of Mauritius. Colon. Geol. Miner. Resour., 1, 217-238. 
Smith, A.G., 1971: Alpine deformation and the oceanic areas of the Tethys, Mediterranean, and Atlantic. Geol. Soc. Am. BuZl., 82, 2039-2070.

Spencer, J.W.W., 1902: On the geological and physical development of Dominica, with notes on Martinique, St. Lucia, St. Vincent, and the Grenadines. Q.J. Geol. Soc. London., 58, 341-353.

Standard, J.C., 1963: Geology of Lord Howe Island. J. Proc. Roy. Soc. N.S.W. 96, 107-121.

Stanton, R.L., 1961: Explanatory notes to accompany a first geological map of Santa Ysabe1, British Solomon Islands Protectorate. Overseas Geol. Miner. Resour., 8, $127-149$.

Stearns, H.T., 1944: Geology of the Samoan Islands. Geol. Soc. Am. Bulz., 55, $1279-1332$.

1945: Late geologic history of the Pacific basin. Am. J. Sci., 243, $614-626$

Stephenson, P.J., 1963: Some geological observations on Heard Island. S.C.A.R. Proc. Antarctic Geol., 14-24.

Thomson, A.A., and Evison, F.F., 1962: Thickness of the earth's crust in New Zealand. N.Z.J. Geol. Geophys., $\underline{5}, 29-45$.

Tracey, J.I., Jr., Sch1anger, S.O., Stark, J.T., Doan, D.B., and May, H.G., 1964 : General geology of Guam. U.S. Geol. Sum. Prof. Pap., 403-A, 1-104.

Umbgrove, J.H.F., 1938: Geological history of the East Indies: BuZ2. Am. Assoc. Pet. Geol. 22, 1-70.

Cambridge, $63 \mathrm{pp}$.

1949: STRUCTURAL HISTORY OF THE EAST INDIES. The University Press,

The Univ. of Sydney, Dept., Geol. and Geophys., 1957 (1956): Gereral reconnaissance of parts of the central islands of the British Solomon Islands Protectorate. Colon. Geol. Miner. Resour., 6, 267-306.

Van Balgooy, M.M.J., 1969: A study on the diversity of island floras. Blumea, 17, $139-178$.

Vogt, P.R., Higgs, R.H., and Johnson, G.L. 1971: Hypotheses on the origin of the Mediterranean Basin: magnetic data. J. Geophys. Res., 76, 3207-3228.

$\overline{810-817}$ , and Ostenso, N.A., 1967: Steady state crustal spreading. Nature 215,

Wa1lace, A.R., 1880: ISLAND LIFE. MacMillan, London, $526 \mathrm{pp}$.

, 1887: Oceanic islands: Their physical and biological relations. Am. Geogr. Soc. BuZZ. N.Y., 19, 1-21.

Wasserburg, G.J., Craig, H., Menard, H.W., Engel, A.E.J., and Engel, C.G., 1963: Age and composition of a Bounty Islands granite and age of a Seychelles Is lands granite. J. Geol., 71, 785-789. 
Waterhouse, J.B., 1971: Permian of New Caledonia. N. \%. J. Geol. Geophys., 14., 249-250.

Weaver, D.W., [1969]: Geology of the Northern Channel Islands. Am. Assoc. Petrol. Geol., Pacif. Sect. Bakersfield, $200 \mathrm{pp}$.

Wentworth, C.K., and Ladd, H.S., 1931: Pacific islands sediments. Univ. Iowa Stud. Nat. Hist., 13, 1-47.

Westermann, J.H., and Kie1, H., 1961: The geology of Saba and St. Eustatius. Uitgaben Natuutwet. Stud. Suriname, Ned. Antilien, 24, 1-175, 33 p1s.

Wey1, R., 1966: Geologie der Antillen. Beitr. Reg. Geol. Erde, 4, 1-410.

Wilhelm, 0., and Ewing, M., 1972: Geology and history of the Gulf of Mexico. Geo?. Soc. Am. Buzl., 83, 575-600.

Williams, H., 1933: Geology of Tahiti, Moorea, and Maiao, BulZ. Bemice P. Bishop Mus. 105, 1-89.

Wi1son, J.T., 1963: A resume of the Geology of Islands in the Main Ocean Basins, 3 vols. Univ. Toronto, Inst. Earth Sei., Sci. Rept. no. 4.

Wood, B.L., 1967: Geology of the Cook Islands. N. Z. J. Geol. Geophys., 10, 1429-1445.

, and Hay, R.F. 1970: Geology of the Cook Islands. N. Z. Geot. Surv. BuzZ., $82, \quad 1-103$.

Woolnough, W.G., 1903: The continental origin of Fiji. Proc. Linn. Soc. N.S.W. 28, 457-540.

Wright, C.W., 1963: Cretaceous ammonites from Bathurst Island, northern Australia. palaeontology, 6, 597-614.

Wright, J.B., and McCurry, P., 1970: The significance of sandstone inclusions in lavas of the Comores Archipelago, M.F.J. Flower and D.F. Strong. Farth Planet. Sci. Lett., 8, 267-268.

Wright, R., 1965: Ramifications of extreme age of St. Peter and St. Paul Rocks. Buzz. Am. Assoc. Pet. Geol. 49, 1709-1711. 
